\title{
SSGP TOPOLOGIES ON ABELIAN GROUPS OF POSITIVE FINITE DIVISIBLE RANK
}

\author{
DMITRI SHAKHMATOV AND VÍCTOR HUGO YAÑEZ
}

In memory of W. Wistar Comfort

\begin{abstract}
For a subset $A$ of a group $G$, we denote by $\langle A\rangle$ the smallest subgroup of $G$ containing $A$ and let $\operatorname{Cyc}(A)=\{x \in G:\langle x\rangle \subseteq A\}$. A topological group $G$ is $S S G P$ if $\langle$ Cyc $(U)\rangle$ is dense in $G$ for every neighbourhood $U$ of zero of $G$. SSGP groups form a proper subclass of the class of minimally almost periodic groups.

Comfort and Gould asked for a characterization of abelian groups which admit an SSGP group topology. An "almost complete" characterization was found by Dikranjan and the first author. The remaining case is resolved here. As a corollary, we give a positive answer to another question of Comfort and Gould by showing that if an abelian group admits an $\operatorname{SSGP}(n)$ group topology for some positive integer $n$, then it admits an SSGP group topology as well.
\end{abstract}

As usual, $\mathbb{Z}$ and $\mathbb{Q}$ denote the groups of integer numbers and rational numbers respectively, $\mathbb{N}$ denotes the set of natural numbers and $\mathbb{N}^{+}=\mathbb{N} \backslash\{0\}$. We use $\mathbf{P}$ to denote the set of prime numbers.

Let $G$ be a group. For subsets $A, B$ of $G$, we let $A B=\{a b: a \in A, b \in B\}$ and $A^{-1}=\left\{a^{-1}\right.$ : $a \in A\}$. When $G$ is abelian, we use the additive notation $A+B$ instead of $A B$ and $-A$ instead of $A^{-1}$. For a subset $A$ of $G$, we denote by $\langle A\rangle$ the smallest subgroup of $G$ containing $A$. To simplify the notation, we write $\langle x\rangle$ instead of $\langle\{x\}\rangle$ for $x \in G$.

\section{The SMALl SUbGroup Generating PROPERTy SSGP}

Following [4], we define

$$
\operatorname{Cyc}(A)=\{x \in G:\langle x\rangle \subseteq A\} \text { for every } A \subseteq G .
$$

Definition 1.1. A topological group $G$ has the small subgroup generating property (abbreviated to $S S G P$ ) if and only if $\langle\mathrm{Cyc}(U)\rangle$ is dense in $G$ for every neighbourhood $U$ of the identity of $G$. We shall say that a topological group $G$ is $S S G P$ if $G$ satisfies the small subgroup generating property.

Remark 1.2. The small subgroup generating property was introduced by Gould [7]; see also [8, 1]. He says that a topological group $G$ has the small subgroup generating property if for every neighbourhood $U$ of the identity of $G$, there exists a family $\mathcal{H}$ of subgroups of $G$ such that $\bigcup \mathcal{H} \subseteq U$ and $\langle\bigcup \mathcal{H}\rangle$ is dense in $G$. It is easily seen that Gould's definition is equivalent to the one given in 1.1 .

Definition 1.3. For a subset $A$ of group $G$ and $k \in \mathbb{N}^{+}$, we let

$$
\langle A\rangle_{k}=\left\{\prod_{i=1}^{j} a_{i}: j \leq k, a_{1}, \ldots, a_{j} \in A\right\} .
$$

2010 Mathematics Subject Classification. Primary: 22A05; Secondary: 03E99, 06A06, 20K27, 54E35, 54H11.

Key words and phrases. minimal almost periodic group, small subgroup generating property, metric group topology, finite powers of the rational numbers, countable group, abelian group, partially ordered set.

The first listed author was partially supported by the Grant-in-Aid for Scientific Research (C) No. 26400091 of the Japan Society for the Promotion of Science (JSPS).

This paper was written as part of the second listed author's studies at the Graduate School of Science and Engineering of Ehime University. The second listed author was partially supported by the 2016 fiscal year grant of the Matsuyama Saibikai. 
Remark 1.4. If $A$ is a subset of a group $G$ such that $A=A^{-1}$, then $\langle A\rangle=\bigcup_{k \in \mathbb{N}^{+}}\langle A\rangle_{k}$.

Using the notation from (2), one can get a convenient reformulation of the SSGP property.

Proposition 1.5. For a topological group $G$, the following conditions are equivalent:

(i) $G$ has the small subgroup generating property SSGP;

(ii) $G=\bigcup_{k \in \mathbb{N}^{+}} W \cdot\langle\operatorname{Cyc}(W)\rangle_{k}$ for every neighbourhood $W$ of the identity of $G$;

(iii) for each $g \in G$ and every neighbourhood $W$ of the identity of $G$, there exist $k \in \mathbb{N}^{+}$ (depending on $g$ and $W$ ), $g_{0} \in W$ and $g_{1}, \ldots, g_{k} \in G$ such that $g=\prod_{i=0}^{k} g_{i}$ and $\left\langle g_{i}\right\rangle \subseteq W$ for $i=1, \ldots, k$.

Proof. (i) $\rightarrow$ (ii) Suppose that $G$ has the small subgroup generating property. Let $W$ be a neighbourhood of the identity $e_{G}$ of $G$. Since $\operatorname{Cyc}(W)=(\operatorname{Cyc}(W))^{-1}$, we have $\langle\operatorname{Cyc}(W)\rangle=\bigcup_{k \in \mathbb{N}^{+}}\langle\operatorname{Cyc}(W)\rangle_{k}$ by Remark 1.4. Furthermore, $\langle\mathrm{Cyc}(W)\rangle$ is dense in $G$ by Definition 1.1, so

$$
G=W \cdot\langle\operatorname{Cyc}(W)\rangle=W \cdot \bigcup_{k \in \mathbb{N}^{+}}\langle\operatorname{Cyc}(W)\rangle_{k}=\bigcup_{k \in \mathbb{N}^{+}} W \cdot\langle\operatorname{Cyc}(W)\rangle_{k} .
$$

(ii) $\rightarrow$ (i) Suppose that $G$ satisfies the property from item (ii) of our lemma. Fix a neighbourhood $U$ of $e_{G}$ in $G$. By Definition 1.1, to check that $G$ has the small subgroup generating property, it suffices to prove that $\langle\mathrm{Cyc}(U)\rangle$ is dense in $G$. This is equivalent to establishing the equality $G=V \cdot\langle\operatorname{Cyc}(U)\rangle$ for every neighbourhood $V$ of $e_{G}$ in $G$. Let $V$ be an arbitrary neighbourhood of $e_{G}$ in $G$. Then $W=U \cap V$ is a neighbourhood of $e_{G}$ in $G$, so we can apply (ii) to this $W$ to obtain

$$
G=\bigcup_{k \in \mathbb{N}^{+}} W \cdot\langle\operatorname{Cyc}(W)\rangle_{k}=W \cdot \bigcup_{k \in \mathbb{N}^{+}}\langle\operatorname{Cyc}(W)\rangle_{k} \subseteq V \cdot \bigcup_{k \in \mathbb{N}^{+}}\langle\operatorname{Cyc}(U)\rangle_{k} \subseteq V \cdot\langle\operatorname{Cyc}(U)\rangle .
$$

The converse inclusion $V \cdot\langle\operatorname{Cyc}(U)\rangle \subseteq G$ is clear.

(ii) $\leftrightarrow$ (iii) follows from (11) and (2).

\section{Properties $\operatorname{SSGP}(n)$ And $\operatorname{SSgP}(\alpha)$}

According to von Neumann's terminology [9], a topological group $G$ is called minimally almost periodic if every continuous homomorphism from $G$ to a compact group is trivial.

For every $n \in \mathbb{N}$, the property $\operatorname{SSGP}(n)$ of topological groups was defined in [7]. We refer the reader to [1, Definition 3.3] for the definition of $\operatorname{SSGP}(n)$. It was proved in [1, Remark 3.4, Theorem 3.5] that the following implications hold for an arbitrary topological group:

(3) $\quad \mathrm{SSGP}=\operatorname{SSGP}(1) \rightarrow \operatorname{SSGP}(2) \rightarrow \ldots \rightarrow \operatorname{SSGP}(n) \rightarrow \ldots \rightarrow$ minimally almost periodic.

Examples distinguishing all properties in (3) can be found in [1, Corollary 3.14, Theorem 4.6]. However, all properties in (3) coincide for bounded torsion abelian topological groups.

Theorem 2.1. [1, Corollary 2.23] A bounded torsion abelian topological group has the small subgroup generating property SSGP if and only if it is minimally almost periodic.

For every ordinal $\alpha$, the property $\operatorname{SSGP}(\alpha)$ of topological groups was defined in [4, Definition 2.3]. The reader is alerted to a subtle difference in fonts used for distinguishing properties $\operatorname{SSGP}(n)$ and $\operatorname{SSGP}(n)$, for $n \in \mathbb{N}$. The connection between these properties is given in items (i), (iv) and (v) of the following theorem.

Theorem 2.2. $\quad$ (i) $\operatorname{SSGP}(1)$ coincides with $\operatorname{SSGP}$ (which is equivalent to $\operatorname{SSGP}(1)$ by (3));

(ii) $\operatorname{SSGP}(\alpha) \rightarrow \operatorname{SSGP}(\beta)$ whenever $\alpha, \beta$ are ordinals satisfying $\alpha<\beta$;

(iii) for each ordinal $\alpha, \operatorname{SSGP}(\alpha) \rightarrow$ minimally almost periodic;

(iv) $\operatorname{SSGP}(n) \rightarrow \operatorname{SSGP}(n)$ for every $n \in \mathbb{N}^{+}$;

(v) for every $n \in \mathbb{N}^{+}$, an abelian topological group has the property $\operatorname{SSGP}(n)$ if and only if it has the property $\operatorname{SSGP}(n)$. 
Proof. Item (i) is [4, Lemma 2.2 and Equation 7], item (ii) is [4, Proposition 5.1], item (iii) is [4, Proposition 5.3 (iii)], item (iv) is [4, Corollary 6.3] and item (v) is [4, Theorem 6.4].

It is unknown if the properties $\operatorname{SSGP}(n)$ and $\operatorname{SSGP}(n)$ coincide for arbitrary topological groups [4, Question 13.4].

\section{INTRODUCTION}

Definition 3.1. We shall say that a topology $\mathscr{T}$ on a group $G$ is an $S S G P$ topology provided that $(G, \mathscr{T})$ is a topological group with the property SSGP. Similar terminology will be used for properties $\operatorname{SSGP}(n)$ for $n \in \mathbb{N}^{+}$and $\operatorname{SSGP}(\alpha)$ for an ordinal $\alpha$.

A characterization of abelian groups admitting a minimal almost periodic group topology is obtained in [5].

Comfort and Gould [1, Questions 5.2 and 5.3] asked the following question.

Question 3.2. (a) What are the (abelian) groups which admit an SSGP topology?

(b) Does every abelian group which for some $n>1$ admits an $\operatorname{SSGP}(n)$ topology also admit an SSGP topology?

Following [6], for an abelian group $G$, we denote by $r_{0}(G)$ the free rank of $G$, by $r_{p}(G)$ the $p$-rank of $G$ for a prime number $p$, and we let

$$
r(G)=\max \left\{r_{0}(G), \sum\left\{r_{p}(G): p \in \mathbf{P}\right\}\right\}
$$

where $\mathbf{P}$ denotes the set of prime numbers.

Definition 3.3. [3, Definition 7.2] For an abelian group $G$, the cardinal

$$
r_{d}(G)=\min \left\{r(n G): n \in \mathbb{N}^{+}\right\}
$$

is called the divisible rank of $G$.

The notion of the divisible rank was defined, under the name of final rank, by Szele [10] for p-groups.

Remark 3.4. An abelian group $G$ satisfies $r_{d}(G)=0$ if and only if $G$ is a bounded torsion group; that is, if $n G=\{0\}$ for some $n \in \mathbb{N}^{+}$.

In view of Remark 3.4, the next theorem coincides with [4, Corollary 1.7].

Theorem 3.5. A non-trivial abelian group $G$ satisfying $r_{d}(G)=0$ admits an SSGP topology if and only if all leading Ulm-Kaplanski invariants of $G$ are infinite.

Theorem 3.5 resolves Question 3.2 (a) for abelian groups of divisible rank zero, while the next theorem resolves it for abelian groups of infinite divisible rank.

Theorem 3.6. [4, Theorem 3.2] Every abelian group $G$ satisfying $r_{d}(G) \geq \omega$ admits an SSGP topology.

In [4], Dikranjan and the first author reduced the remaining case $0<r_{d}(G)<\omega$ to a question regarding the existence of SSGP topologies on abelian groups of a very particular type. More precisely, assuming a positive answer to their question, formulated in [4] as Question 13.1, they established in [4, Theorem 13.2] what is stated below as Theorem 4.1, thus giving a (provisional, at that moment) complete characterization of abelian groups $G$ admitting an SSGP topology in the remaining open case $0<r_{d}(G)<\omega$. 


\section{Results}

The main goal of the paper is to provide a positive answer to (a more general version of) [4, Question 13.1] given in Theorem 9.2 below. It follows from this theorem that [4, Theorem 13.2] holds unconditionally; that is, the following result holds:

Theorem 4.1. For an abelian group $G$ satisfying $1 \leq r_{d}(G)<\omega$, the following conditions are equivalent:

(i) $G$ admits an SSGP topology;

(ii) $G$ admits an $\operatorname{SSGP}(\alpha)$ topology for some ordinal $\alpha$;

(iii) the quotient $H=G / t(G)$ of $G$ with respect to its torsion part $t(G)$ has finite rank $r_{0}(H)$ and $r(H / A)=\omega$ for some (equivalently, every) free subgroup $A$ of $H$ such that $H / A$ is torsion.

A combination of Theorems 3.5, 3.6 and 4.1 provides a complete solution to item (a) of Question 3.2 for abelian groups. Similarly, the implication (iii) $\rightarrow$ (i) of the next corollary provides a complete solution to item (b) of the same question for abelian groups. Both items of Question 3.2 remain widely open for non-commutative groups.

Corollary 4.2. For an abelian group $G$, the following conditions are equivalent:

(i) $G$ admits an SSGP topology;

(ii) $G$ admits an $\operatorname{SSGP}(\alpha)$ topology for some ordinal $\alpha$;

(iii) $G$ admits an $\operatorname{SSGP}(n)$ topology for some integer $n>1$.

Proof. The implication (i) $\rightarrow$ (iii) follows from (3), while the implication (iii) $\rightarrow$ (ii) follows from item (iv) of Theorem 2.2 .

(ii) $\rightarrow$ (i) Let $G$ be an abelian $\operatorname{SSGP}(\alpha)$ group for some ordinal $\alpha$. We consider three cases.

Case 1. $r_{d}(G)=0$. By Remark 3.4, $G$ is a bounded torsion group. Since $G$ is an $\operatorname{SSGP}(\alpha)$ group, it is minimally almost periodic by item (iii) of Theorem 2.2, so $G$ is SSGP by Theorem 2.1.

Case 2. $1 \leq r_{d}(G)<\omega$. In this case $G$ admits an SSGP topology by the implication (ii) $\rightarrow(\mathrm{i})$ of Theorem 4.1 .

Case 3. $r_{d}(G) \geq \omega$. In this case $G$ admits an SSGP topology by Theorem 3.6.

The paper is organized as follows. In Section 5 we prove two lemmas about the algebraic structure of subgroups $\mathbb{Q}_{\pi}^{m}$ of finite powers $\mathbb{Q}^{m}$ of $\mathbb{Q}$ which are needed in the proof of the main theorem. In Section $\left[6\right.$ we introduce the notion of a wide subgroup $G$ of $\mathbb{Q}^{m}$ and we establish two auxiliary lemmas about wide subgroups needed in the sequel. The main result of the paper is Theorem 9.2 which states that a direct sum $G \oplus H$ of a wide subgroup $G$ of $\mathbb{Q}^{m}$ and at most countable abelian group $H$ admits a metric SSGP topology $\mathscr{T}$. Let us explain the strategy for constructing this topology. First, we introduce a poset $(\mathbb{P}, \leq)$ in Section 7 . Next, we define a countable family $\mathscr{D}$ of dense subsets of $(\mathbb{P}, \leq)$ and select a linearly ordered subset $\mathbb{F}$ of $(\mathbb{P}, \leq)$ intersecting all members of the family $\mathscr{D}$; this can be done due to a folklore Lemma 9.1. Finally, the countable base of neighbourhoods of zero for $\mathscr{T}$ is defined by means of elements of $\mathbb{F}$ in Section 9 . The family $\mathscr{D}$ is defined in (35). Section 8 collects lemmas establishing density in $(\mathbb{P}, \leq)$ of various sets participating in the family $\mathscr{D}$. The "technical heart" of this section is Lemma 8.6 which is responsible for the SSGP property of $\mathscr{T}$.

The reader familiar with Martin's Axiom undoubtedly notices that we are using here a "ZFC version" of this axiom when the family of dense sets is at most countable. The choice of such an exposition was determined by the author's desire to replace a direct construction of $\mathscr{T}$ via an induction (which would be totally incomprehensible) by a "much smoother" forcing-type argument using a poset $(\mathbb{P}, \leq)$ and some dense subsets of it (which is much easier to follow than the direct inductive construction). We hope that, after discovering the technical complexity even of this "smooth" approach, the reader would fully agree with our judgment. 


\section{The algebraic structure of subgroups $\mathbb{Q}_{\pi}^{m}$ of $\mathbb{Q}^{m}$}

Definition 5.1. For a non-empty set $\pi$ of of prime numbers, we use $\mathbb{Q}_{\pi}$ to denote the set of all rational numbers $q$ whose irreducible representation $q=z / n$ with $z \in \mathbb{Z}$ and $n \in \mathbb{N}^{+}$is such that all prime divisors of $n$ belong to $\pi$. For convenience, we let $\mathbb{Q}_{\emptyset}=\{0\}$.

Definition 5.2. Given an integer $s \in \mathbb{Z}$, we shall denote by $\lceil s\rceil$ the subgroup $s \mathbb{Z}$ of $\mathbb{Q}$.

A straightforward proof of the following lemma is left to the reader.

Lemma 5.3. Let $\pi \subseteq \mathbf{P}$ and $m \in \mathbb{N}^{+}$. Then:

(i) $\mathbb{Q}_{\pi}$ is a subgroup of $\mathbb{Q}$, so $\mathbb{Q}_{\pi}^{m}$ is a subgroup of $\mathbb{Q}^{m}$.

(ii) $\mathbb{Z} \subseteq \mathbb{Q}_{\pi}$, and so $\lceil s\rceil^{m} \subseteq \mathbb{Q}_{\pi}^{m}$ for each $s \in \mathbb{N}^{+}$.

(iii) If $\pi \subseteq \pi^{\prime} \subseteq \mathbf{P}$, then $\mathbb{Q}_{\pi} \subseteq \mathbb{Q}_{\pi^{\prime}}$, and so $\mathbb{Q}_{\pi}^{m} \subseteq \mathbb{Q}_{\pi^{\prime}}^{m}$.

Our next lemma clarifies the algebraic structure of subgroups $\mathbb{Q}_{\pi}^{m}$ of $\mathbb{Q}^{m}$.

Lemma 5.4. (A) Suppose that $s \in \mathbb{Z}, k \in \mathbb{N}^{+}, g_{1}, \ldots, g_{k} \in \mathbb{Q}^{m}, \pi_{0}, \pi_{1}, \ldots, \pi_{k} \in[\mathbf{P}]^{<\omega}$,

$$
\pi_{0} \subseteq \pi_{1} \subseteq \pi_{2} \subseteq \cdots \subseteq \pi_{k}
$$

and the following conditions hold for every $j=1,2, \ldots, k$ :

$\left(\mathrm{a}_{j}\right) g_{j} \in \mathbb{Q}_{\pi_{j}}^{m}$,

$\left(\mathrm{b}_{j}\right)\left\langle g_{j}\right\rangle \cap \mathbb{Q}_{\pi_{j-1}}^{m} \subseteq\lceil s\rceil^{m}$.

Then:

(i) $\left\langle\left\{g_{1}, \ldots, g_{i}\right\}\right\rangle+\mathbb{Q}_{\pi_{0}}^{m} \subseteq \mathbb{Q}_{\pi_{i}}^{m}$ for every $i=1, \ldots, k$.

(ii) $\left\langle\left\{g_{i}, \ldots, g_{k}\right\}\right\rangle \cap \mathbb{Q}_{\pi_{i-1}}^{m} \subseteq\lceil s\rceil^{m}$ for every $i=1, \ldots, k$.

(B) In addition to the assumptions of item (A), suppose that the following condition holds for every $j=1,2, \ldots, k$ :

$\left(\mathrm{c}_{j}\right) m g_{j} \notin \mathbb{Q}_{\pi_{j-1}}^{m}$ for every $m \in \mathbb{Z} \backslash\{0\}$ satisfying $|m| \leq k$.

Finally, assume that $g \in \mathbb{Q}_{\pi_{0}}^{m}, J$ is a proper subset of the set $\{1,2, \ldots, k\}, l \in \mathbb{Z},|l| \leq k$ and

$$
l g_{0} \in\left\langle\left\{g_{j}: j \in J\right\}\right\rangle+\mathbb{Q}_{\pi_{0}}^{m},
$$

where

$$
g_{0}=g-\sum_{j=1}^{k} g_{j}
$$

Then $l=0$.

Proof. First, we check item (A).

(i) Let $i=1,2, \ldots, k$ be arbitrary. Fix $j=1, \ldots, i$. Then $\pi_{j} \subseteq \pi_{i}$ by (15). Applying Lemma 5.3 (iii), we get $\mathbb{Q}_{\pi_{j}}^{m} \subseteq \mathbb{Q}_{\pi_{i}}^{m}$. Since $g_{j} \in \mathbb{Q}_{\pi_{j}}^{m}$ by $\left(\mathrm{a}_{j}\right)$, we obtain $g_{j} \in \mathbb{Q}_{\pi_{i}}^{m}$. Since this holds for arbitrary $j=1, \ldots, i$, we conclude that $\left\{g_{1}, \ldots, g_{i}\right\} \subseteq \mathbb{Q}_{\pi_{i}}^{m}$. Since $\pi_{0} \subseteq \pi_{i}$ by (5) , applying Lemma 5.3 (iii) once again, we obtain that $\mathbb{Q}_{\pi_{0}}^{m} \subseteq \mathbb{Q}_{\pi_{i}}^{m}$. Since $\mathbb{Q}_{\pi_{i}}^{m}$ is a subgroup of $\mathbb{Q}^{m}$ by Lemma 5.3 (i), this implies $\left\langle\left\{g_{1}, \ldots, g_{i}\right\}\right\rangle+\mathbb{Q}_{\pi_{0}}^{m} \subseteq \mathbb{Q}_{\pi_{i}}^{m}$.

(ii) We shall prove item (ii) using induction on $k$.

Basis of induction. If $k=1$, then the conclusion of item (ii) holds by ( $\mathrm{b}_{1}$ ).

Inductive step. Let $k \geq 2$ and suppose that item (ii) has already been proved for $k-1$.

Let $i=1, \ldots, k$ by arbitrary. Fix

$$
h \in\left\langle\left\{g_{i}, \ldots, g_{k}\right\}\right\rangle \cap \mathbb{Q}_{\pi_{i-1}}^{m} .
$$

Then there exist $x \in\left\langle\left\{g_{i}, \ldots, g_{k-1}\right\}\right\rangle$ and $y \in\left\langle g_{k}\right\rangle$ such that $h=x+y$. In particular, $x \in \mathbb{Q}_{\pi_{k-1}}^{m}$ by item (i), as $0 \in \mathbb{Q}_{\pi_{0}}^{m}$. Since $i-1 \leq k-1$, we have $\pi_{i-1} \subseteq \pi_{k-1}$ by (15), which implies $\mathbb{Q}_{\pi_{i-1}}^{m} \subseteq \mathbb{Q}_{\pi_{k-1}}^{m}$ by 
Lemma 5.3 (iii). From this and (8), we obtain $h \in \mathbb{Q}_{\pi_{k-1}}^{m}$. Therefore, $y=h-x \in \mathbb{Q}_{\pi_{k-1}}^{m}-\mathbb{Q}_{\pi_{k-1}}^{m}=$ $\mathbb{Q}_{\pi_{k-1}}^{m}$, as $\mathbb{Q}_{\pi_{k-1}}^{m}$ is a subgroup of $\mathbb{Q}^{m}$. Now $y \in\left\langle g_{k}\right\rangle \cap \mathbb{Q}_{\pi_{k-1}}^{m} \subseteq\lceil s\rceil^{m}$ by $\left(\mathrm{b}_{k}\right)$. Recalling item (ii) of Lemma [5.3, we conclude that $y \in \mathbb{Q}_{\pi_{i-1}}^{m}$. Since $h \in \mathbb{Q}_{\pi_{i}}^{m}$ by (죠), we get $x=h-y \in \mathbb{Q}_{\pi_{i-1}}^{m}-\mathbb{Q}_{\pi_{i-1}}^{m}=$ $\mathbb{Q}_{\pi_{i-1}}^{m}$, as $\mathbb{Q}_{\pi_{i-1}}^{m}$ is a subgroup of $\mathbb{Q}^{m}$. We obtained that $x \in\left\langle\left\{g_{i}, \ldots, g_{k-1}\right\}\right\rangle \cap \mathbb{Q}_{\pi_{i-1}}^{m}$. By our inductive assumption, $\left\langle\left\{g_{i}, \ldots, g_{k-1}\right\}\right\rangle \cap \mathbb{Q}_{\pi_{i-1}}^{m} \subseteq\lceil s\rceil^{m}$, so $x \in\lceil s\rceil^{m}$. Since $y \in\lceil s\rceil^{m}$ as well, $h=x+y \in\lceil s\rceil^{m}+\lceil s\rceil^{m}=\lceil s\rceil^{m}$.

Next, we check item (B). From (6) and (7), we get

$$
l g-\sum_{j=1}^{k} l g_{j} \in\left\langle\left\{g_{j}: j \in J\right\}\right\rangle+\mathbb{Q}_{\pi_{0}}^{m} .
$$

Since $\mathbb{Q}_{\pi_{0}}^{m}$ is a subgroup of $\mathbb{Q}^{m}$ by Lemma 5.3 (i) and $g \in \mathbb{Q}_{\pi_{0}}^{m}$ by our assumption, it follows that

$$
-\sum_{j=1}^{k} l g_{j} \in\left\langle\left\{g_{j}: j \in J\right\}\right\rangle+\mathbb{Q}_{\pi_{0}}^{m} .
$$

Since $J$ is a proper subset of the set $\{1,2, \ldots, k\}$, we can fix $i \in\{1,2, \ldots, k\} \backslash J$. From this and (9), we can find

$$
x \in\left\langle\left\{g_{1}, \ldots, g_{i-1}\right\}\right\rangle \text { and } y \in\left\langle\left\{g_{i+1}, \ldots, g_{k}\right\}\right\rangle
$$

such that

$$
l g_{i} \in x+y+\mathbb{Q}_{\pi_{0}}^{m} .
$$

(If $i=1$, we define $x=0$, and if $i=k$, we define $y=0$.) Then

$$
y \in l g_{i}-x+\mathbb{Q}_{\pi_{0}}^{m} \in\left\langle g_{i}\right\rangle-\left\langle\left\{g_{1}, \ldots, g_{i-1}\right\}\right\rangle+\mathbb{Q}_{\pi_{0}}^{m}=\left\langle\left\{g_{1}, \ldots, g_{i}\right\}\right\rangle+\mathbb{Q}_{\pi_{0}}^{m} \subseteq \mathbb{Q}_{\pi_{i}}^{m}
$$

by subitem (i) of item (A). Recalling the second inclusion in (10), we get $y \in\left\langle\left\{g_{i+1}, \ldots, g_{k}\right\}\right\rangle \cap \mathbb{Q}_{\pi_{i}}^{m}$. Now applying subitem (ii) of item (A), we conclude that $y \in\lceil s\rceil^{m}$. Combining this with Lemma 5.3 (ii), we get $y \in \mathbb{Q}_{\pi_{i-1}}^{m}$. From the first inclusion in (10) and subitem (i) of item (A), we get $x \in \mathbb{Q}_{\pi_{i}}^{m}$. Note that $\mathbb{Q}_{\pi_{0}}^{m} \subseteq \mathbb{Q}_{\pi_{i-1}}^{m}$ by subitem (i) of item (A), as $0 \in\left\langle\left\{g_{1}, \ldots g g_{i}\right\}\right\rangle$. Since $\mathbb{Q}_{\pi_{i-1}}^{m}$ is a subgroup of $\mathbb{Q}^{m}$, from $x, y \in \mathbb{Q}_{\pi_{i-1}}^{m}$, the inclusion $\mathbb{Q}_{\pi_{0}}^{m} \subseteq \mathbb{Q}_{\pi_{i-1}}^{m}$ and (11), one obtains $l g_{i} \in \mathbb{Q}_{\pi_{i-1}}^{m}$. Since $l \in \mathbb{Z}$ and $|l| \leq k$, applying $\left(\mathrm{c}_{i}\right)$ with $m=l$, we get $l=0$.

\section{Wide Subgroups of $\mathbb{Q}^{m}$}

Our next definition gives a name to subgroups of $\mathbb{Q}^{m}$ having the property from [4, Question 13.1].

Definition 6.1. Let $m \in \mathbb{N}^{+}$. We shall call a subgroup $G$ of $\mathbb{Q}^{m}$ wide if $\mathbb{Z}^{m} \subseteq G$ and $G \backslash \mathbb{Q}_{\pi}^{m} \neq \emptyset$ for every $\pi \in[\mathbf{P}]^{<\omega}$.

Lemma 6.2. Let $G$ be a wide subgroup of $\mathbb{Q}^{m}, \pi \in[\mathbf{P}]^{<\omega}, k \in \mathbb{N} \backslash\{0\}$ and $s \in \mathbb{Z} \backslash\{0\}$. Then there exists $g \in G$ such that:

(i) $\langle g\rangle \cap \mathbb{Q}_{\pi}^{m} \subseteq\lceil s\rceil^{m}$,

(ii) $l g \notin \mathbb{Q}_{\pi}^{m}$ for every $l \in \mathbb{Z} \backslash\{0\}$ satisfying $|l| \leq k$.

Proof. Let $\varpi$ be the set of all prime numbers not exceeding $\max \{k, s\}$. Then $\pi^{\prime}=\pi \cup \varpi \in[\mathbf{P}]^{<\omega}$. Since $G$ is nice, we can find $h \in G \backslash \mathbb{Q}_{\pi^{\prime}}^{m}$. Let $h=\left(h_{1}, \ldots, h_{m}\right)$, where $h_{1}, \ldots, h_{m} \in \mathbb{Q}$. For every $i=1, \ldots, m$, let $h_{i}=a_{i} / b_{i}$ be the irreducible fraction with $a_{i} \in \mathbb{Z}$ and $b_{i} \in \mathbb{N}^{+}$.

Since $h \notin \mathbb{Q}_{\pi^{\prime}}^{m}$, we can fix $t \in\{1, \ldots, m\}$ such that $h_{t} \notin \mathbb{Q}_{\pi^{\prime}}$. Since $a_{t} / b_{t}$ is an irreducible representation of $h_{t} \in \mathbb{Q} \backslash \mathbb{Q}_{\pi^{\prime}}$, we can fix $p \in \mathbf{P} \backslash \pi^{\prime}$ dividing $b_{t}$. Since $\varpi \subseteq \pi^{\prime}$ and $p \in \mathbf{P} \backslash \pi^{\prime}$, we have $p \in \mathbf{P} \backslash \varpi$. Since $\varpi$ includes all prime numbers not exceeding $\max \{k, s\}$, we conclude that

$$
p>\max \{k, s\} \text {. }
$$


For every $i=1, \ldots, m$, let

$$
n_{i}=\max \left\{n \in \mathbb{N}: p^{n} \text { divides } b_{i}\right\}
$$

Then

$$
c_{i}=b_{i} / p^{n_{i}} \in \mathbb{N} \text { is not divisible by } p .
$$

Note that

$$
n_{t} \geq 1
$$

as $p$ divides $b_{t}$ by our choice of $p$. It follows from $s \in \mathbb{N}$ and (14) that

$$
m_{0}=s c_{1} c_{2} \cdots c_{m} \in \mathbb{N} .
$$

Since $G$ is a group and $h \in G$, it follows that $g=m_{0} h \in G$. Note that

$$
g=\left(m_{0} h_{1}, \ldots, m_{0} h_{m}\right) .
$$

We claim that $g$ is the required element of $G$; that is, conditions (i) and (ii) are satisfied.

Fix $i=1, \ldots, m$. It follows from (14) and (16) that

$$
m_{0} h_{i}=s c_{1} c_{2} \cdots c_{m} \frac{a_{i}}{b_{i}}=s\left(\prod_{j=1, j \neq i}^{m} c_{j}\right) \frac{b_{i}}{p^{n_{i}}} \frac{a_{i}}{b_{i}}=s\left(\prod_{j=1, j \neq i}^{m} c_{j}\right) \frac{a_{i}}{p^{n_{i}}}=\frac{s d_{i}}{p^{n_{i}}},
$$

where

$$
d_{i}=c_{1} \cdots c_{i-1} a_{i} c_{i+1} \cdots c_{m} \in \mathbb{Z} .
$$

Claim 1. The right-hand side of (18) is the irreducible representation of $m_{0} h_{i}$.

Proof. If $n_{i}=0$, then $p^{n_{i}}=1$, so $s d_{i} / 1$ is the irreducible representation of $m_{0} h_{i}$. Suppose now that $n_{i} \geq 1$. In this case, it suffices to check that neither $s$ nor $d_{i}$ is divisible by $p$. The first statement follows from (12). Since $n_{i} \geq 1$, (13) implies that $p$ divides $b_{i}$. Since $a_{i} / b_{i}$ is an irreducible fraction, $p$ does not divide $a_{i}$. By (14), each $c_{j}$ is not divisible by $p$. By (19), this means that $p$ does not divide $d_{i}$.

(i) By (17), in order to check (i), we need to show that $\left\langle m_{0} h_{i}\right\rangle \cap \mathbb{Q}_{\pi} \subseteq s \mathbb{Z}$ for every $i=1, \ldots, m$. Fix such an integer $i$. Let $x \in\left\langle m_{0} h_{i}\right\rangle \cap \mathbb{Q}_{\pi}$. Then $x=l m_{0} h_{i}$ for some $l \in \mathbb{Z}$. Since $p \notin \pi$, it follows from $x \in \mathbb{Q}_{\pi}$, Definition $\left[5.1\right.$ and Claim 1 that $p^{n_{i}}$ must divide $l$, so $l=p^{n_{i}} l^{\prime}$ for some $l^{\prime} \in \mathbb{Z}$. Therefore, $x=l m_{0} h_{i}=l^{\prime} s d_{i}$ by (18). Since $l^{\prime} \in \mathbb{Z}$ and $d_{i} \in \mathbb{Z}$ by (19), we conclude that $x \in\lceil s\rceil$. This implies $\left\langle m_{0} h_{i}\right\rangle \cap \mathbb{Q}_{\pi} \subseteq\lceil s\rceil$.

(ii) By (17), in order to check (ii), it suffices to show that $l m_{0} h_{t} \notin \mathbb{Q}_{\pi}^{m}$ for every $l \in \mathbb{Z} \backslash\{0\}$ satisfying $|l| \leq k$. Fix $l$ satisfying these conditions. Since $|l| \leq k<p$ by (12), $p$ does not divide $l$. Recalling Claim 1 and (18), we conclude that $l s d_{t} / p^{n_{t}}$ is the irreducible representation of $l m_{0} h_{t}$. Since $p \notin \pi$ and $n_{t} \geq 1$ by (15), from this and Definition [5.1, we obtain that $l m_{0} h_{t} \notin \mathbb{Q}_{\pi}$.

Lemma 6.3. Let $m \in \mathbb{N}^{+}, G$ be a wide subgroup of $\mathbb{Q}^{m}, \pi_{0} \in[\mathbf{P}]^{<\omega}, k \in \mathbb{N}^{+}$and $s \in \mathbb{Z} \backslash\{0\}$. Then there exist an increasing sequence $\pi_{0} \subseteq \pi_{1} \subseteq \pi_{2} \subseteq \cdots \subseteq \pi_{k}$ of finite subsets of $\mathbf{P}$ and elements $g_{1}, \ldots, g_{k} \in G$ such that conditions $\left(a_{j}\right),\left(b_{j}\right),\left(c_{j}\right)$ of Lemma 5.4 hold for every $j=1, \ldots, k$.

Proof. The lemma is proved by finite induction on $k \in \mathbb{N}^{+}$based on Lemma 6.2. 


\section{The poset $(\mathbb{P}, \leq)$}

In this section we define a (technically rather involved) poset $(\mathbb{P}, \leq)$ which shall be used to construct a metric SSGP topology $\mathscr{T}$ on the direct sum $G \oplus H$ of a wide subgroup of $\mathbb{Q}^{m}$ for $m \in \mathbb{N}^{+}$and an arbitrary at most countable abelian group $H$. The definition of a poset itself does not require the group $G$ is to be wide and the countability restriction on $H$ is not essential, so we do not impose either of these two conditions in the next definition.

Definition 7.1. Let $H$ be an abelian group. For a fixed $m \in \mathbb{N}^{+}$, consider the direct sum $\mathbb{Q}^{m} \oplus H$. Furthermore, let $G$ be a subgroup of $\mathbb{Q}^{m}$ containing $\mathbb{Z}^{m}$. Then the sum $G+H=G \oplus H$ is direct.

(a) Let $\mathbb{P}$ be the set of all structures $p=\ll \pi^{p}, n^{p},\left\{U_{i}^{p}: i \in n^{p}+1\right\},\left\{s_{i}^{p}: i \in n^{p}+1\right\} \gg$ satisfying the following conditions:

$\left(1_{p}\right) \pi^{p} \in[\mathbf{P}]^{<\omega}$,

$\left(2_{p}\right) n^{p} \in \mathbb{N}$,

$\left(3_{p}\right) s_{i}^{p} \in \mathbb{N}^{+}$for every $i \in n^{p}+1$,

$\left(4_{p}\right) 0 \in U_{i}^{p} \subseteq\left(G \cap \mathbb{Q}_{\pi^{p}}^{m}\right)+H$ for every $i \in n^{p}+1$,

$\left(5_{p}\right)-U_{i}^{p}=U_{i}^{p}$ for every $i \in n^{p}+1$,

$\left(6_{p}\right) U_{i}^{p}+\left\lceil s_{i}^{p}\right\rceil^{m}=U_{i}^{p}$ for every $i \in n^{p}+1$,

$\left(7_{p}\right) U_{i+1}^{p}+U_{i+1}^{p} \subseteq U_{i}^{p}$ for every $i \in n^{p}$

$\left(8_{p}\right) s_{i}^{p}$ divides $s_{i+1}^{p}$ for every $i \in n^{p}$.

(b) Given structures

$$
p=\ll \pi^{p}, n^{p},\left\{U_{i}^{p}: i \in n^{p}+1\right\},\left\{s_{i}^{p}: i \in n^{p}+1\right\} \gg \in \mathbb{P}
$$

and

$$
q=\ll \pi^{q}, n^{q},\left\{U_{i}^{q}: i \in n^{q}+1\right\},\left\{s_{i}^{q}: i \in n^{q}+1\right\} \gg \in \mathbb{P},
$$

we define $q \leq p$ if and only if the following conditions are satisfied:

$\left(\mathrm{i}_{q}^{p}\right) \pi^{p} \subseteq \pi^{q}$

$\left(\mathrm{ii}_{q}^{p}\right) n^{p} \leq n^{q}$

$\left(\operatorname{iii}_{q}^{p}\right) U_{i}^{q} \cap\left(\mathbb{Q}_{\pi^{p}}^{m}+H\right)=U_{i}^{p}$ for every $i \in n^{p}+1$,

$\left(\operatorname{iv}_{q}^{p}\right) s_{i}^{q}=s_{i}^{p}$ for every $i \in n^{p}+1$.

Remark 7.2. Let $p=\ll \pi^{p}, n^{p},\left\{U_{i}^{p}: i \in n^{p}+1\right\},\left\{s_{i}^{p}: i \in n^{p}+1\right\},\left\{s_{i}^{p}: i \in n^{p}+1\right\} \gg \in \mathbb{P}$. Then:

(i) $U_{i+1}^{p} \subseteq U_{i}^{p}$ for every $i \in n^{p}$. Indeed, $0 \in U_{i+1}^{p}$ by $\left(4_{p}\right)$, so $U_{i+1}^{p}=0+U_{i+1}^{p} \subseteq U_{i+1}^{p}+U_{i+1}^{p} \subseteq U_{i}^{p}$ by $\left(7_{p}\right)$.

(ii) $\left\lceil s_{i}^{p}\right\rceil^{m} \subseteq U_{i}^{p}$ for every $i \in n^{p}+1$. Indeed, $U_{i}^{p}+\left\lceil s_{i}^{p}\right\rceil^{m}=U_{i}^{p}$ by $\left(6_{p}\right)$. Since $0 \in U_{i}^{p}$ by $\left(4_{p}\right)$, this implies $\left\lceil s_{i}^{p}\right\rceil^{m} \subseteq U_{i}^{p}$.

A straightforward proof of the following lemma is left to the reader.

Lemma 7.3. ( $\mathbb{P}, \leq)$ is a poset.

Lemma 7.4. $\mathbb{P} \neq \emptyset$.

Proof. Define $\pi^{p}=\emptyset, n^{p}=0, s_{0}^{p}=1, U_{0}^{p}=\mathbb{Z}^{m}$, and

$$
p=\ll \pi^{p}, n^{p},\left\{U_{i}^{p}: i \in 1\right\},\left\{s_{i}^{p}: i \in 1\right\} \gg=\ll \emptyset, 0,\left\{U_{0}^{p}\right\},\left\{s_{0}^{p}\right\} \gg .
$$

To show that $p \in \mathbb{P}$, we need to check the conditions $\left(1_{p}\right)-\left(8_{p}\right)$ of Definition 7.1 (a). Conditions $\left(1_{p}\right)-\left(3_{p}\right)$ are clear, and conditions $\left(7_{p}\right)$ and $\left(8_{p}\right)$ are vacuous. Conditions $\left(5_{p}\right)$ and $\left(6_{p}\right)$ are satisfied, as $U_{0}^{p}=\mathbb{Z}^{m}$ is a subgroup of $\mathbb{Q}^{m}$ and $\lceil 1\rceil^{m}=\mathbb{Z}^{m}$.

Lemma 7.5. Given $p \in \mathbb{P}, g \in G \cap \mathbb{Q}_{\pi_{p}}^{m}$ and $h \in H$ with $g+h \neq 0$, one can find $q \in \mathbb{P}$ such that $q \leq p, n^{q}=n^{p}+1$ and $g+h \notin U_{n^{q}}$.

Proof. Let 
- $\pi^{q}=\pi^{p}$

- $n^{q}=n^{p}+1$,

- $U_{i}^{q}=U_{i}^{p}$ and $s_{i}^{q}=s_{i}^{p}$ for every $i \in n^{p}+1$.

It remains only to define $U_{n^{q}}^{q}$ and $s_{n^{q}}^{q}$.

Since $\bigcap_{k \in \mathbb{N}^{+}}\left\lceil k s_{n^{p}}^{p}\right\rceil^{m}=\{0\}$ and $g+h \neq 0$, there exists $k \in \mathbb{N}^{+}$such that $g+h \notin\left\lceil k s_{n^{p}}^{p}\right\rceil^{m}$. Define $s_{n_{q}}^{q}=k s_{n^{p}}^{p}$. Since $s_{n^{p}}^{p} \in \mathbb{N}^{+}$by $\left(3_{p}\right)$ and $k \in \mathbb{N}^{+}, s_{n_{q}}^{q} \in \mathbb{N}^{+}$and

$$
s_{n^{p}}^{p} \text { divides } s_{n^{q}}^{q} \text {. }
$$

By our choice of $s_{n_{q}}^{q}$, we have

$$
g+h \notin\left\lceil s_{n^{q}}^{q}\right\rceil^{m} .
$$

Finally, we define

$$
U_{n^{q}}^{q}=\left\lceil s_{n^{q}}^{q}\right\rceil^{m}
$$

Claim 2. $q=\ll \pi^{q}, n^{q},\left\{U_{i}^{q}: i \in n^{q}+1\right\},\left\{s_{i}^{q}: i \in n^{q}+1\right\} \gg \in \mathbb{P}$.

Proof. According to Definition 7.1 (a), we have to check that the structure $q$ satisfies conditions $\left(1_{q}\right)-\left(8_{q}\right)$. By our construction, conditions $\left(1_{q}\right),\left(2_{q}\right),\left(3_{q}\right)$ and $\left(8_{q}\right)$ hold.

Let us check conditions $\left(4_{q}\right)-\left(7_{q}\right)$. Since conditions $\left(4_{p}\right)-\left(7_{p}\right)$ hold and $U_{i}^{q}=U_{i}^{p}, s_{i}^{q}=s_{i}^{p}$ for every $i \in n^{p}+1$, it follows that conditions $\left(4_{q}\right)-\left(6_{q}\right)$ hold for each $i \in n^{p}+1=n^{q}$ and condition $\left(7_{q}\right)$ holds for each $i \in n^{p}$. Therefore, it remains only to check the following four conditions:

(a) $0 \in U_{n^{q}}^{q} \subseteq\left(G \cap \mathbb{Q}_{\pi^{q}}^{m}\right)+H$,

(b) $-U_{n^{q}}^{q}=U_{n^{q}}^{q}$

(c) $U_{n^{q}}^{q}+\left\lceil s_{n^{q}}^{q}\right\rceil^{m}=U_{n^{q}}^{q}$,

(d) $U_{n_{q}}^{q}+U_{n_{q}}^{q} \subseteq U_{n_{p}}^{q}$.

Conditions (b) and (c) are immediate from (22) and the fact that $\left\lceil s_{n^{q}}^{q}\right\rceil^{m}$ is a subgroup of $\mathbb{Q}^{m}$.

Clearly, $0 \in\left\lceil s_{n^{q}}^{q}\right\rceil^{m} \subseteq \mathbb{Z}^{m} \subseteq G$ by our assumption on $G$. Furthermore, $\left\lceil s_{n^{q}}^{q}\right\rceil^{m} \subseteq \mathbb{Q}_{\pi^{q}}^{q}$ by Lemma 5.3 (ii). Combining this with (22), we get $0 \in U_{n_{q}}^{q} \subseteq G \cap \mathbb{Q}_{\pi^{q}}^{m} \subseteq\left(G \cap \mathbb{Q}_{\pi^{q}}^{m}\right)+H$. Thus, (a) holds.

From (22), we get $U_{n_{q}}^{q}+U_{n_{q}}^{q}=\left\lceil s_{n^{q}}^{q}\right\rceil^{m}+\left\lceil s_{n^{q}}^{q}\right\rceil^{m}=\left\lceil s_{n^{q}}^{q}\right\rceil^{m}$. Since $s_{n^{p}}^{p}$ divides $s_{n^{q}}^{q}$ by (20), we have the inclusion $\left\lceil s_{n^{q}}^{q}\right\rceil^{m} \subseteq\left\lceil s_{n^{p}}^{p}\right\rceil^{m}$. Finally, $\left\lceil s_{n^{p}}^{p}\right\rceil^{m} \subseteq U_{n_{p}}^{q}$ by Remark $\mathbf{7 . 2}$ (ii). This finishes the check of $(d)$.

The inequality $q \leq p$ is clear from our construction of $q$ and Definition 7.1 (b). Finally, from (21) and (22), we get $g+h \notin U_{n^{q}}$.

\section{Density LemMas}

Definition 8.1. Let $(\mathbb{P}, \leq)$ be a poset. Recall that a set $D \subseteq \mathbb{P}$ is called:

(i) dense in $(\mathbb{P}, \leq)$ provided that for every $p \in \mathbb{P}$ there exists $q \in D$ such that $q \leq p$;

(ii) downward-closed in $(\mathbb{P}, \leq)$ if for every $p \in D$ and $q \in \mathbb{P}$ the inequality $q \leq p$ implies that $q \in D$.

The relation between these two notions is made apparent by the following straightforward lemma.

Lemma 8.2. If $A, B \subseteq \mathbb{P}$ are dense subsets of a poset $(\mathbb{P}, \leq)$ and $A$ is downward-closed, then $A \cap B$ is dense in $(\mathbb{P}, \leq)$.

Lemma 8.3. For every $n \in \mathbb{N}$, the set $A_{n}=\left\{q \in \mathbb{P}: n \leq n^{q}\right\}$ is dense and downward-closed in $(\mathbb{P}, \leq)$.

Proof. Let $n \in \mathbb{N}$ and $p \in \mathbb{P}$. If $n \leq n^{p}$, then $p \in A_{n}$, so we shall assume from now on that $n^{p}<n$. Then $k=n-n^{p} \geq 1$.

Note that $\mathbb{Z}^{m} \subseteq G$ by our assumption on $G$ and $\mathbb{Z}^{m} \subseteq \mathbb{Q}_{\pi^{p}}^{m}$ by Lemma 5.3 (ii), so $\mathbb{Z}^{m} \subseteq G \cap \mathbb{Q}_{\pi^{p}}^{m}$. This allows us to fix $g \in G \cap \mathbb{Q}_{\pi^{p}}^{m}$ with $g \neq 0$. Let $h=0$. Then $g+h=g \neq 0$. 
Let $q_{0}=p$. By finite induction on $i=1, \ldots, k$, we can use Lemma 7.5 to find $q_{i} \in \mathbb{P}$ such that $q_{i} \leq q_{i-1}$ and $n^{q_{i}}=n^{q_{i-1}}+1=n^{p}+i$. (Note that $g$ and $h$ play a "dummy role" in this argument; their sole purpose here is to make the assumptions of Lemma 7.5 satisfied.) Now $q_{k} \leq q_{k-1} \leq \cdots \leq q_{1} \leq q_{0}=p$ and $n^{q_{k}}=n^{p}+k=n$, so $q_{k} \in A_{n}$. This shows that $A_{n}$ is dense in $(\mathbb{P}, \leq)$.

Finally, given $p \in A_{n}$ and $q \in \mathbb{P}$ such that $q \leq p$, we have that $n \leq n^{p} \leq n^{q}$, thus showing that $q \in A_{n}$ and therefore, that $A_{n}$ is downward-closed.

Lemma 8.4. For every $\pi \in[\mathbf{P}]^{<\omega}$, the set $B_{\pi}=\left\{q \in \mathbb{P}: \pi \subseteq \pi^{q}\right\}$ is dense in $(\mathbb{P}, \leq)$.

Proof. Let $\pi \in[\mathbf{P}]^{<\omega}$ and $p \in \mathbb{P}$. Define

- $\pi^{q}=\pi^{p} \cup \pi$,

- $n^{q}=n^{p}$,

- $s_{i}^{q}=s_{i}^{p}$ for every $i \in n^{p}+1$; and

- $U_{i}^{q}=U_{i}^{p}$ for every $i \in n^{p}+1$.

A straightforward check using Definition 7.1 (a) shows that

$$
q=\ll \pi^{q}, n^{q},\left\{U_{i}^{q}: i \in n^{q}+1\right\},\left\{s_{i}^{q}: i \in n^{q}+1\right\} \gg \in \mathbb{P} .
$$

From our definition of $q$ and Definition 7.1 (b), one easily concludes that $q \leq p$. Clearly, $q \in B_{\pi}$. This shows that $B_{\pi}$ is dense in $(\mathbb{P}, \leq)$.

Lemma 8.5. The set $C_{g+h}=\left\{q \in \mathbb{P}: g+h \in\left(\mathbb{Q}_{\pi^{q}}^{m}+H\right) \backslash U_{n^{q}}^{q}\right\}$ is dense in $(\mathbb{P}, \leq)$ whenever $g \in G$, $h \in H$ and $g+h \neq 0$.

Proof. Fix $g \in G$ and $h \in H$ such that $g+h \neq 0$. Let $r \in \mathbb{P}$ be arbitrary. Since $g \in G \subseteq \mathbb{Q}^{m}$, there exists $\pi \in[\mathbf{P}]^{<\omega}$ such that $g \in \mathbb{Q}_{\pi}^{m}$. Since $B_{\pi}$ is dense in $(\mathbb{P}, \leq)$ by Lemma 8.4 there exists $p \in B_{\pi}$ such that $p \leq r$. Now $\pi \subseteq \pi^{p}$ by definition of $B_{\pi}$, so $\mathbb{Q}_{\pi}^{m} \subseteq \mathbb{Q}_{\pi^{p}}^{m}$ by Lemma 5.3 (iii). Since $g \in \mathbb{Q}_{\pi}^{m}$, we also have $g \in \mathbb{Q}_{\pi^{p}}^{m}$. We have checked that $g \in G \cap \mathbb{Q}_{\pi^{p}}^{m}$. Applying Lemma 7.5, we can find $q \in \mathbb{P}$ such that $q \leq p \leq r$ and $g+h \notin U_{n^{q}}$. Since $q \leq p$, we have $\pi^{p} \subseteq \pi^{q}$ by $\left(\mathrm{i}_{q}^{p}\right)$, so $\mathbb{Q}_{\pi^{p}}^{m} \subseteq \mathbb{Q}_{\pi^{q}}^{m}$ by Lemma $\left[5.3\right.$ (iii). Since $g \in \mathbb{Q}_{\pi^{p}}^{m}$, we also have $g \in \mathbb{Q}_{\pi^{q}}^{m}$. Therefore, $g+h \in \mathbb{Q}_{\pi^{q}}^{m}+H$. We have proved that $g+h \in\left(\mathbb{Q}_{\pi^{q}}^{m}+H\right) \backslash U_{n^{q}}^{q}$, so $q \in C_{g+h}$. Since $q \leq r$, this shows that $C_{g+h}$ is dense in $(\mathbb{P}, \leq)$.

Lemma 8.6. If $G$ is a wide subgroup of $\mathbb{Q}^{m}$, then the set

$$
D_{g+h}=\left\{q \in \mathbb{P}: g+h \in \bigcup_{k \in \mathbb{N}^{+}} U_{n^{q}}^{q}+\left\langle\operatorname{Cyc}\left(U_{n^{q}}^{q}\right)\right\rangle_{k}\right\}
$$

is dense in $(\mathbb{P}, \leq)$ for all $g \in G$ and $h \in H$.

Proof. Let $g \in G, h \in H$ and $p \in \mathbb{P}$ be arbitrary. By Lemma 8.4, we may assume, without loss of generality, that $g \in \mathbb{Q}_{\pi^{p}}$. Define

$$
k=2^{n^{p}}+1
$$

Applying Lemma 6.3 to $\pi_{0}=\pi^{p}$ and $s=s_{n^{p}}^{p}$, we can find an increasing sequence

$$
\pi^{p}=\pi_{0} \subseteq \pi_{1} \subseteq \pi_{2} \subseteq \cdots \subseteq \pi_{k}=\pi^{q}
$$

of finite subsets of $\mathbf{P}$ and elements $g_{1}, \ldots, g_{k} \in G$ such that conditions $\left(\mathrm{a}_{j}\right),\left(\mathrm{b}_{j}\right),\left(\mathrm{c}_{j}\right)$ of Lemma 5.4 (in which we let $s=s_{n^{p}}^{p}$ ) hold for every $j=1, \ldots, k$.

Define $g_{0}$ as in (7). Since $G$ is a subgroup of $\mathbb{Q}, g \in \mathbb{Q}_{\pi_{0}}$ and $\left(\mathrm{a}_{j}\right)$ holds for every $j=1, \ldots, k$, it follows from (5) and (7) that

$$
\left\{g_{j}: j \in k+1\right\} \subseteq \mathbb{Q}_{\pi^{q}}^{m}
$$


Let

$$
U_{n^{p}}^{q}=U_{n^{p}}^{p} \cup\left(\left(\left\{g_{0}+h\right\} \cup\left\{-\left(g_{0}+h\right)\right\} \cup \bigcup_{j=1}^{k}\left\langle g_{j}\right\rangle\right)+\left\lceil s_{n^{p}}^{p}\right\rceil^{m}\right) .
$$

By finite reverse induction, we define

$$
U_{i}^{q}=U_{i}^{p} \cup\left(U_{i+1}^{q}+U_{i+1}^{q}+\left\lceil s_{i}^{p}\right\rceil^{m}\right)
$$

for every $i=n^{p}-1, \ldots, 0$.

Let $n^{q}=n^{p}$ and $s_{i}^{q}=s_{i}^{p}$ for every $i \in n^{q}+1=n^{p}+1$.

Claim 3. $q=\ll \pi^{q}, n^{q},\left\{U_{i}^{q}: i \in n^{q}+1\right\},\left\{s_{i}^{q}: i \in n^{q}+1\right\} \gg \in \mathbb{P}$.

Proof. We need to check conditions $\left(1_{q}\right)-\left(8_{q}\right)$.

Conditions $\left(1_{q}\right),\left(2_{q}\right)$ and $\left(3_{q}\right)$ are trivial.

$\left(4_{q}\right)$ By $\left(4_{p}\right)$ and (28), $0 \in U_{i}^{p} \subseteq U_{i}^{q}$ for every $i \in n^{q}+1=n^{p}+1$. By $\left(4_{p}\right), U_{i}^{p} \subseteq\left(G \cap \mathbb{Q}_{\pi^{p}}^{m}\right)+H$ for every $i \in n^{q}+1=n^{p}+1$. Since $G$ is a subgroup of $\mathbb{Q}^{m}$, from this, $g_{1}, \ldots, g_{k} \in G$ and (27), it follows that $U_{n^{q}}^{q}=U_{n^{p}}^{q} \subseteq G+H$. Furthermore, (26) and (27) imply that $U_{n^{q}}^{q}=U_{n^{p}}^{q} \subseteq \mathbb{Q}_{\pi_{q}}^{m}+H$. Thus, $U_{n^{q}}^{q} \subseteq\left(G \cap \mathbb{Q}_{\pi^{q}}^{m}\right)+H$. Since $\left(G \cap \mathbb{Q}_{\pi^{q}}^{m}\right)+H$ is a subgroup of $\mathbb{Q}^{m}+H$, from this, (28) and finite reverse induction on $i=n^{q}, n^{q}-1, \ldots, 0$, one concludes that $U_{i}^{q} \subseteq\left(G \cap \mathbb{Q}_{\pi^{q}}^{m}\right)+H$ for every $i=n^{q}+1$.

$\left(5_{q}\right)$ From (27) and $\left(5_{p}\right)$, we get $-U_{n^{p}}=U_{n^{p}}$. Starting with this and using (28), $\left(5_{p}\right)$ and finite reverse induction on $i=n^{q}, n^{q}-1, \ldots, 0$, one concludes that $-U_{i}^{q}=U_{i}^{q}$ for every $i=n^{q}+1$.

$\left(6_{q}\right)$ The equation $U_{n^{p}}^{q}+\left\lceil s_{n^{p}}^{p}\right\rceil^{m}=U_{n^{p}}^{q}$ follows from (27) and $\left(6_{p}\right)$. Starting with this and using (28),,$\left(6_{p}\right)$ and finite reverse induction on $i=n^{p}, n^{p}-1, \ldots, 0$, one concludes that $U_{i}^{q}+\left\lceil s_{i}^{p}\right\rceil^{m}=U_{i}^{q}$ for every $i=n^{p}+1$. Since $n^{q}=n^{p}$ and $s_{i}^{q}=s_{i}^{p}$ for every $i \in n^{q}+1$, this establishes $\left(6_{q}\right)$.

$\left(7_{q}\right)$ follows from (28).

$\left(8_{q}\right)$ follows from $\left(8_{p}\right)$, as $s_{i}^{q}=s_{i}^{p}$ for every $i \in n^{q}+1$.

Claim 4. $q \in D_{g+h}$.

Proof. It follows from (27) that $g_{0}+h \in U_{n^{q}}^{q}$ and $g_{1}, \ldots, g_{k} \in \operatorname{Cyc}\left(U_{n^{q}}^{q}\right)$. Furthermore, $g=$ $g_{0}+\sum_{i=1}^{k} g_{i}$ by (7). Thus,

$$
g+h=g_{0}+h+\sum_{i=1}^{k} g_{i} \in U_{n^{q}}^{q}+\left\langle\operatorname{Cyc}\left(U_{n^{q}}^{q}\right)\right\rangle_{k}
$$

by Definition 1.3, so $q \in D_{g+h}$ by (23).

Our final goal is to prove the inequality $q \leq p$. Before establishing this, we need to check two auxiliary facts.

Claim 5. The inclusion

$$
U_{i}^{q} \subseteq \bigcup\left\{U_{i}^{p}+l\left(g_{0}+h\right)+\sum_{j \in J}\left\langle g_{j}\right\rangle+\left\lceil s_{i}^{p}\right\rceil^{m}: l \in \mathbb{Z},|l| \leq 2^{n^{q}-i}, J \in\{1,2, \ldots, k\}^{\leq 2^{n^{q}-i}}\right\}
$$

holds for every $i \in n^{p}+1$.

Proof. This is proved by reverse induction on $i=n^{p}, n^{p}-1, \ldots, 0$. For $i=n^{p}$ this follows from (27). 
Let us make an inductive step. Suppose that $i \in n^{p}$ and we have already proved the inclusion (29) for $i+1$; that is, we have shown that the inclusion

$$
U_{i+1}^{q} \subseteq \bigcup\left\{U_{i+1}^{p}+l\left(g_{0}+h\right)+\sum_{j \in J}\left\langle g_{j}\right\rangle+\left\lceil s_{i+1}^{p}\right\rceil^{m}: l \in \mathbb{Z},|l| \leq 2^{n^{q}-i-1}, J \in\{1,2, \ldots, k\} \leq 2^{n^{q}-i-1}\right\}
$$

holds. We are going to show that the inclusion (29) holds as well.

Let $x \in U_{i}^{q}$. By equation (28), either $x \in U_{i}^{p}$ or $x \in U_{i+1}^{q}+U_{i+1}^{q}+\left\lceil s_{i}^{p}\right\rceil^{m}$. In the former case, $x=x+0\left(g_{0}+h\right)+0+0$ is the decomposition witnessing that $x$ belongs to the right-hand side of (29). In the latter case, $x=y_{0}+y_{1}+s_{i}^{p} w$, where $w \in \mathbb{Z}^{m}$ and $y_{t} \in U_{i+1}^{q}$ for $t=0,1$. Applying (30), we can find $u_{t} \in U_{i+1}^{p}, l_{t} \in \mathbb{Z}, J_{t} \in\{1,2, \ldots, k\}^{\leq 2^{n^{q}-i-1}}, b_{t} \in \sum_{j \in J_{t}}\left\langle g_{j}\right\rangle$ and $z_{t} \in \mathbb{Z}^{m}$ for $t=0,1$ such that $\left|l_{t}\right| \leq 2^{n^{q}-i-1}$ and $y_{t}=u_{t}+l_{t}\left(g_{0}+h\right)+b_{t}+s_{i+1}^{p} z_{t}$. Since $x=y_{0}+y_{1}+s_{i}^{p} w$, it follows that $x=u+l\left(g_{0}+h\right)+b+s_{i+1}^{p} z+s_{i}^{p} w$, where $u=u_{0}+u_{1}, l=l_{0}+l_{1}, b=b_{0}+b_{1}$ and $z=z_{0}+z_{1}$. Since $s_{i}^{p}$ divides $s_{i+1}^{p}$ by $\left(8_{p}\right), s_{i+1}^{p}=s_{i}^{p} k_{0}$ for some $k_{0} \in \mathbb{Z}$, so

$$
x=u+l\left(g_{0}+h\right)+b+s_{i}^{p}\left(k_{0} z+w\right) .
$$

Since $u_{t} \in U_{i+1}^{p}$ for $t=0,1, u=u_{0}+u_{1} \in U_{i+1}^{p}+U_{i+1}^{p} \subseteq U_{i}^{p}$ by $\left(7_{p}\right)$. Since $l_{t} \in \mathbb{Z}$ and $\left|l_{t}\right| \leq 2^{n^{q}-i-1}$ for $t=0,1$, we have $l=l_{0}+l_{1} \in \mathbb{Z}$ and

$$
|l| \leq\left|l_{0}\right|+\left|l_{1}\right| \leq 2^{n^{q}-i-1}+2^{n^{q}-i-1}=2 \cdot 2^{n^{q}-i-1}=2^{n^{q}-i} .
$$

Since $J_{t} \in\{1,2, \ldots, k\}^{\leq 2^{n^{q}}-i-1}$ for $t=0,1$, the set $J=J_{0} \cup J_{1}$ satisfies

$$
|J| \leq\left|J_{1}\right|+\left|J_{2}\right| \leq 2^{n^{q}-i-1}+2^{n^{q}-i-1}=2 \cdot 2^{n^{q}-i-1}=2^{n^{q}-i}
$$

so $J \in\{1,2, \ldots, k\}^{\leq 2^{n^{q}-i}}$. Since $b_{t} \in \sum_{j \in J_{t}}\left\langle g_{j}\right\rangle$ for $t=0,1$, we have $b=b_{0}+b_{1} \in \sum_{j \in J}\left\langle g_{j}\right\rangle$. Since $z_{t} \in \mathbb{Z}^{m}$ for $t=0,1$, we have $z=z_{0}+z_{1} \in \mathbb{Z}^{m}$. Finally, since $k_{0} \in \mathbb{Z}$, we get $k_{0} z+w \in \mathbb{Z}^{m}$ and thus $s_{i}^{p}\left(k_{0} z+w\right) \in\left\lceil s_{i}^{p}\right\rceil^{m}$. Combining all this with (31), we conclude that

$$
x \in U_{i}^{p}+l\left(g_{0}+h\right)+\sum_{j \in J}\left\langle g_{j}\right\rangle+\left\lceil s_{i}^{p}\right\rceil^{m}
$$

with $l \in \mathbb{Z}, J \in\{1,2, \ldots, k\} \leq^{2^{q^{q}-i}}$ and $|l| \leq 2^{n^{q}-i}$. This finishes the proof of the inclusion (29).

Claim 6. $U_{i}^{q} \cap\left(\mathbb{Q}_{\pi^{p}}^{m}+H\right)=U_{i}^{p}$ for every $i \in n^{p}+1$.

Proof. It follows from (28) that $U_{i}^{p} \subseteq U_{i}^{q}$. Since $U_{i}^{p} \subseteq \mathbb{Q}_{\pi^{p}}^{m}+H$ by $\left(4_{p}\right)$, this establishes the inclusion $U_{i}^{p} \subseteq U_{i}^{q} \cap\left(\mathbb{Q}_{\pi^{p}}^{m}+H\right)$.

To prove the reverse inclusion, we fix an arbitrary $x \in U_{i}^{q} \cap\left(\mathbb{Q}_{\pi^{p}}^{m}+H\right)$ and we are going to show that $x \in U_{i}^{p}$. Since $x \in U_{i}^{q}$, we can apply Claim 5 to fix $y \in U_{i}^{p}, l \in \mathbb{Z}$, a finite set $J \subseteq\{1,2, \ldots, k\}$, a family $\left\{m_{j}: j \in J\right\} \subseteq \mathbb{Z}$ and $z \in \mathbb{Z}^{m}$ such that $|l| \leq 2^{n^{q}-i},|J| \leq 2^{n^{q}-i}$ and

$$
x=y+l\left(g_{0}+h\right)+\sum_{j \in J} m_{j} g_{j}+s_{i}^{p} z .
$$

Recall that $x \in \mathbb{Q}_{\pi^{p}}^{m}+H$. Furthermore, $y \in U_{i}^{p} \subseteq \mathbb{Q}_{\pi^{p}}^{m}+H$ by $\left(4_{p}\right)$. Finally, $s_{i}^{p} z \in \mathbb{Z}^{m} \subseteq \mathbb{Q}_{\pi^{p}}^{m}$. Since $\pi_{0}=\pi^{p}$ by (25) and $\mathbb{Q}_{\pi_{0}}^{m}$ is a group, from (32) we get

$$
l\left(g_{0}+h\right)+\sum_{j \in J} m_{j} g_{j}=x-y-s_{i}^{p} z \in \mathbb{Q}_{\pi_{0}}^{m}+H,
$$

so

$$
l g_{0}=-h-\sum_{j \in J} m_{j} g_{j}+\mathbb{Q}_{\pi_{0}}^{m}+H \in\left\langle\left\{g_{j}: j \in J\right\}\right\rangle+\mathbb{Q}_{\pi_{0}}^{m}+H .
$$


Since $\left\{g_{0}\right\} \cup\left\{g_{j}: j \in J\right\} \subseteq \mathbb{Q}^{m}, \mathbb{Q}_{\pi_{0}}^{m} \subseteq \mathbb{Q}^{m}, \mathbb{Q}^{m}$ is a group and the sum $\mathbb{Q}^{m}+H=\mathbb{Q}^{m} \oplus H$ is direct, from the inclusion (34) we obtain the stricter inclusion $l g_{0} \in\left\langle\left\{g_{j}: j \in J\right\}\right\rangle+\mathbb{Q}_{\pi_{0}}^{m}$.

Since $|J| \leq 2^{n^{q}-i} \leq 2^{n^{q}}=2^{n^{p}}<k$ by (24), $J$ is a proper subset of the set $\{1,2, \ldots, k\}$. Now all the assumptions of Lemma 5.4 are satisfied (with $s=s_{n^{p}}^{p}$ ), so from item (B) of this lemma we conclude that $l=0$. From this and (33), we obtain $\sum_{j \in J} m_{j} g_{j} \in \mathbb{Q}_{\pi_{0}}^{m}+H$, so we can fix $a \in \mathbb{Q}_{\pi_{0}}^{m}$ and $b \in H$ satisfying $\sum_{j \in J} m_{j} g_{j}=a+b$. Since $\mathbb{Q}_{\pi^{q}}^{m}$ is a subgroup of $\mathbb{Q}^{m}$, from (26) we get $\sum_{j \in J} m_{j} g_{j} \in \mathbb{Q}_{\pi^{q}}^{m}$. Therefore,

$$
b=(-a)+\sum_{j \in J} m_{j} g_{j} \in \mathbb{Q}_{\pi_{0}}^{m}+\mathbb{Q}_{\pi^{q}}^{m} \subseteq \mathbb{Q}^{m} .
$$

Since $b \in H$, we get $b \in \mathbb{Q}^{m} \cap H$. Since the sum $\mathbb{Q}^{m}+H=\mathbb{Q}^{m} \oplus H$ is direct, we conclude that $b=0$. Thus, $\sum_{j \in J} m_{j} g_{j}=a \in \mathbb{Q}_{\pi_{0}}^{m}$. Since $J \subseteq\{1,2, \ldots, k\}$, we get

$$
\sum_{j \in J} m_{j} g_{j} \in\left\langle\left\{g_{j}: j \in J\right\}\right\rangle \cap \mathbb{Q}_{\pi_{0}}^{m} \subseteq\left\langle\left\{g_{1}, g_{2}, \ldots, g_{k}\right\}\right\rangle \cap \mathbb{Q}_{\pi_{0}}^{m} \subseteq\left\lceil s_{n^{p}}^{p}\right\rceil^{m}
$$

by subitem (ii) of item (A) of Lemma 5.4 applied with $s=s_{n^{p}}^{p}$. Combining this with (32), we get $x \in y+\left\lceil s_{n^{p}}^{p}\right\rceil^{m}+\left\lceil s_{i}^{p}\right\rceil^{m}$. Since $i \leq n_{p}, s_{i}^{p}$ divides $s_{n^{p}}^{p}$ by $\left(8_{p}\right)$, so $\left\lceil s_{n^{p}}^{p}\right\rceil^{m} \subseteq\left\lceil s_{i}^{p}\right\rceil^{m}$. This gives $x \in y+\left\lceil s_{i}^{p}\right\rceil^{m} \subseteq U_{i}^{p}+\left\lceil s_{i}^{p}\right\rceil^{m}=U_{i}^{p}$ by $\left(6_{p}\right)$.

Claim 7. $q \leq p$.

Proof. We need to check conditions $\left(\mathrm{i}_{q}^{p}\right)-\left(\mathrm{iv}_{q}^{p}\right)$. ( $\left.\mathrm{i}_{q}^{p}\right)$ follows from (5). Since $n^{q}=n^{p}$ by our definition, $\left(\mathrm{ii}_{q}^{p}\right.$ ) holds. ( $\mathrm{iii}_{q}^{p}$ ) is proved in Claim [6, (iv ${ }_{q}^{p}$ ) holds by the definition of $s_{i}^{q}$.

The density of $D_{g+h}$ in $(\mathbb{P}, \leq)$ follows from Claims 3 , 4 and 7 .

\section{MAIN THEOREM}

We shall need the following folklore lemma.

Lemma 9.1. If $\mathscr{D}$ is an at most countable family of dense subsets of a non-empty poset $(\mathbb{P}, \leq)$, then there exists an at most countable subset $\mathbb{F}$ of $\mathbb{P}$ such that $(\mathbb{F}, \leq)$ is a linearly ordered set and $\mathbb{F} \cap D \neq \emptyset$ for every $D \in \mathscr{D}$.

Proof. Since the family $\mathscr{D}$ is at most countable, we can fix an enumeration $\mathscr{D}=\left\{D_{n}: n \in \mathbb{N}^{+}\right\}$of elements of $\mathscr{D}$. Since $\mathbb{P} \neq \emptyset$, there exists $p_{0} \in \mathbb{P}$. By induction on $n \in \mathbb{N}^{+}$, we can choose $p_{n} \in D_{n}$ such that $p_{n} \leq p_{n-1}$; this is possible because $D_{n}$ is dense in $(\mathbb{P}, \leq)$. Now $\mathbb{F}=\left\{p_{n}: n \in \mathbb{N}^{+}\right\}$is the desired subset of $\mathbb{P}$.

The next theorem provides a positive answer to a more general version of [4, Question 13.1].

Theorem 9.2. Suppose that $m \in \mathbb{N}^{+}$and $G$ is a wide subgroup of $\mathbb{Q}^{m}$. Then for each at most countable abelian group $H$, the direct sum $K=G \oplus H$ admits a metric SSGP topology.

Proof. Fix $m \in \mathbb{N}^{+}$and let $G$ be a wide subgroup of $\mathbb{Q}^{m}$. Let $H$ be a countable abelian group. We work in the direct sum $\mathbb{Q}^{m} \oplus H$ and consider its subgroup $K=G+H$. Clearly, the sum $G+H=G \oplus H$ is direct. Therefore, $K$ can also be considered as the direct product $G \times H$ of $G$ and $H$. This observation means that it suffices to find the required group topology $\mathscr{T}$ on the subgroup $K=G+H$ of the direct sum $\mathbb{Q}^{m} \oplus H$.

Let $(\mathbb{P}, \leq)$ be the poset from Definition 7.1 which uses our $m \in \mathbb{N}^{+}, G$ and $H$ as its parameters.

As a subgroup of $\mathbb{Q}^{m}, G$ is at most countable. Since $H$ is at most countable as well, so is the sum $K=G+H$. Therefore, the family

$$
\mathscr{D}=\left\{C_{x}: x \in K \backslash\{0\}\right\} \cup\left\{A_{n} \cap D_{x}: n \in \mathbb{N}, x \in K\right\}
$$

of subsets of $\mathbb{P}$ is at most countable. 
Lst us check that all members of $\mathscr{D}$ are dense in $(\mathbb{P}, \leq)$. By Lemma 8.5, each $C_{x}$ for $x \in K \backslash\{0\}$ is dense in $(\mathbb{P}, \leq)$. Let $n \in \mathbb{N}$ and $x \in K$. Since $A_{n}$ is dense and downward-closed in $(\mathbb{P}, \leq)$ by Lemma 8.3 and $D_{x}$ is dense in $(\mathbb{P}, \leq)$ by Lemma 8.6. from Lemma 8.2 we conclude that $A_{n} \cap D_{x}$ is dense in $(\mathbb{P}, \leq)$.

Apply Lemma 9.1 to find a countable set $\mathbb{F} \subseteq \mathbb{P}$ such that $(\mathbb{F}, \leq)$ is a linearly ordered set and $\mathbb{F} \cap D \neq \emptyset$ for every $D \in \mathscr{D}$. For every $i \in \mathbb{N}$, define

$$
U_{i}=\bigcup\left\{U_{i}^{p}: p \in \mathbb{F} \text { and } i \leq n^{p}\right\} .
$$

Our nearest goal is to show that the family

$$
\mathcal{B}=\left\{U_{i}: i \in \mathbb{N}\right\}
$$

is a neighbourhood base at 0 of a Hausdorff group topology $\mathscr{T}$ on $K$. The verification of this will be split into three claims.

Claim 8. $\bigcap_{n \in \mathbb{N}} U_{n}=\{0\}$.

Proof. Let $n \in \mathbb{N}$ be arbitrary. Since $A_{n} \cap D_{0} \in \mathscr{D}$ by (35), there exists $p \in A_{n} \cap D_{0} \cap \mathbb{F}$. Therefore, $n \leq n^{p}$ by the definition of $A_{n}$. Now $0 \in U_{n}^{p}$ by the condition $\left(4_{p}\right)$ imposed on $\mathbb{P}$. Since $p \in \mathbb{F}$ and $n \leq n^{p}$, it follows from (36) that $U_{n}^{p} \subseteq U_{n}$. This shows that $0 \in U_{n}$. Since $n \in \mathbb{N}$ was chosen arbitrarily, we conclude that $0 \in \bigcap_{n \in \mathbb{N}} U_{n}$.

To prove the reverse inclusion $\bigcap_{n \in \mathbb{N}} U_{n} \subseteq\{0\}$, we choose $x \in K \backslash\{0\}$ arbitrarily and show that $x \notin \bigcap_{n \in \mathbb{N}} U_{n}$. Since $C_{x} \in \mathscr{D}$ by (35), our choice of $\mathbb{F}$ allows us to fix $p \in C_{x} \cap \mathbb{F}$. Then

$$
x \in\left(\mathbb{Q}_{\pi^{p}}^{m}+H\right) \backslash U_{n^{p}}^{p}
$$

by the definition of $C_{x}$.

Assume that $x \in U_{n^{p}}$. Thus, by (36), there exists $q \in \mathbb{F}$ such that $n^{p} \leq n^{q}$ and $x \in U_{n^{p}}^{q}$. Suppose that $p \leq q$. Then $U_{n^{p}}^{p} \cap\left(\mathbb{Q}_{\pi^{q}}^{m}+H\right)=U_{n^{p}}^{q}$ by $\left(\mathrm{iii}_{p}^{q}\right)$. Since $x \in U_{n^{p}}^{q}$, this implies $x \in U_{n^{p}}^{p}$, in contradiction with (38). Similarly, assume that $q \leq p$. Then $U_{n^{p}}^{q} \cap\left(\mathbb{Q}_{\pi^{p}}^{m}+H\right)=U_{n^{p}}^{p}$ by (iii $q$ ), so $x \notin U_{n^{p}}^{q}$ by (38), in contradiction with $x \in U_{n^{p}}^{q}$. This contradiction shows that neither $p \leq q$ nor $q \leq p$ holds. Since $p, q \in \mathbb{F}$, this contradicts the fact that $\mathbb{F}$ is linearly ordered by $\leq$. This contradiction shows that our assumption that $x \in U_{n^{p}}$ is false, so $x \notin \bigcap_{n \in \mathbb{N}} U_{n}$.

Claim 9. $-U_{i}=U_{i}$ and $U_{i+1}+U_{i+1} \subseteq U_{i}$ for every $i \in \mathbb{N}$.

Proof. Let $x \in U_{i}$. By (36), this means that $x \in U_{i}^{p}$ for some $p \in \mathbb{F}$ satisfying $i \leq n^{p}$. By $\left(5_{p}\right)$, we know that $-U_{i}^{p}=U_{i}^{p}$, so $-x \in U_{i}^{p}$. This proves the inclusion $-U_{i} \subseteq U_{i}$. The reverse inclusion is proved analogously.

Finally, consider $x, y \in U_{i+1}$. By (36), there exist $p, q \in \mathbb{F}$ such that $x \in U_{i+1}^{p}, y \in U_{i+1}^{q}, i+1 \leq n^{p}$ and $i+1 \leq n^{q}$. Since $(\mathbb{F}, \leq)$ is a linear order, we may assume, without loss of generality, that $q \leq p$. Since $i+1 \leq n^{p}$, (iii $\left.q\right)$ implies $x \in U_{i+1}^{p} \subseteq U_{i+1}^{q}$. Therefore, $x, y \in U_{i+1}^{q}$. Since $i+1 \leq n^{q}$, we have $x+y \in U_{i+1}^{q}+U_{i+1}^{q} \subseteq U_{i}^{q}$ by $\left(7_{q}\right)$. Then $x+y \in U_{i}$ by (36). This shows that $U_{i+1}+U_{i+1} \subseteq U_{i}$, as desired.

Claim 10. The family $\mathcal{B}$ as in (37) is a neighbourhood base at 0 of some Hausdorff group topology $\mathscr{T}$ on $K$.

Proof. It easily follows from Claims 8 and 9 that $U_{m} \subseteq U_{n}$ whenever $n, m \in \mathbb{N}$ and $n \leq m$. Combined with (37), this implies that $\mathcal{B}$ is a filter base. By (37) and Claim 9 , $\mathcal{B}$ has the following two properties.

- For every $U \in \mathcal{B}$ there exists $V \in \mathcal{B}$ such that $V+V \subseteq U$; and

- For every $U \in \mathcal{B}$ there exists $V \in \mathcal{B}$ such that $-V \subseteq U$. 
By [2, Theorem 3.1.5], the family

$$
\mathscr{T}=\{O \subseteq K: \forall a \in O \exists U \in \mathcal{B}(a+U \subseteq O)\}
$$

is a topology on $K$ making it into a topological group such that the family $\mathcal{B}$ is a neighbourhood base at 0 comprised of $\mathscr{T}$-neighbourhoods of 0 . It follows from Claim 8 and [2, Theorem 4.1.1] that $\mathscr{T}$ is Hausdorff.

Claim 11. The topological group $(K, \mathscr{T})$ has the small subgroup generating property.

Proof. We are going to check that $(K, \mathscr{T})$ has the property (ii) from Proposition 1.5 .

Let $W$ be a neighbourhood of 0 in $(K, \mathscr{T})$. By Claim 10, there exists $i \in \mathbb{N}$ such that $U_{i} \subseteq W$.

Take an arbitrary $x \in K$. Since $A_{i} \cap D_{x} \in \mathscr{D}$, there exists $q \in \mathbb{F} \cap A_{i} \cap D_{x}$. Since $q \in D_{x}$, we can find $k \in \mathbb{N}$ such that

$$
x \in U_{n_{q}}^{q}+\left\langle\operatorname{Cyc}\left(U_{n_{q}}^{q}\right)\right\rangle_{k} .
$$

Since $q \in A_{i}$, we have $i \leq n^{q}$, so $U_{n^{q}}^{q} \subseteq U_{i}^{q}$ by Remark 7.2 (i). Since $q \in \mathbb{F}$, from (36) we get $U_{i}^{q} \subseteq U_{i}$, so $U_{n^{q}}^{q} \subseteq U_{i} \subseteq W$, which implies $\operatorname{Cyc}\left(U_{n^{q}}^{q}\right) \subseteq \operatorname{Cyc}(W)$. Therefore,

$$
U_{n_{q}}^{q}+\left\langle\operatorname{Cyc}\left(U_{n_{q}}^{q}\right)\right\rangle_{k} \subseteq W+\langle\operatorname{Cyc}(W)\rangle_{k} .
$$

Combining (39) and (40), we conclude that $x \in \bigcup_{k \in \mathbb{N}^{+}} W+\langle\operatorname{Cyc}(W)\rangle_{k}$. Since this inclusion holds for every $x \in K$, we get $K \subseteq \bigcup_{k \in \mathbb{N}^{+}} W+\langle\operatorname{Cyc}(W)\rangle_{k}$. The converse inclusion is clear.

Since $(K, \mathscr{T})$ is Hausdorff and has a countable base at 0 by Claim 10, it is metrizable.

\section{REFERENCES}

[1] W.W. Comfort and F. R. Gould, Some classes of minimally almost periodic topological groups, Appl. Gen. Topol. 16 (2015), 141-165.

[2] D. Dikranjan, Introduction to Topological Groups, Reference Notes, University of Udine, 2013.

[3] D. Dikranjan and D. Shakhmatov, A complete solution of Markov's problem on connected group topologies, Adv. Math. 286 (2016), 286-307.

[4] D. Dikranjan and D. Shakhmatov, Topological groups with many small subgroups, Topology Appl. 200 (2016), 101-132.

[5] D. Dikranjan and D. Shakhmatov, Final solution of Protasov-Comfort's problem on minimally almost periodic group topologies, preprint, arXiv:1410.3313.

[6] L. Fuchs, Infinite Abelian groups, Vol. I, Academic Press, New York, 1970.

[7] F. Gould, On certain classes of minimally almost periodic groups, Thesis (Ph.D.), Wesleyan University. 2009. 136 pp. ISBN: 978-1109-22005-6.

[8] F. Gould, An SSGP topology for $\mathbb{Z}^{\omega}$, Topology Proc. 44 (2014), 389-392.

[9] J. von Neumann, Almost periodic functions in a group I, Trans. Amer. Math. Soc. 36 (1934), $445-492$.

[10] T. Szele, On the basic subgroups of abelian p-groups, Acta Math. Acad. Sci. Hungar. 5 (1954), $129-141$.

Division of Mathematics, Physics and Earth Sciences, Graduate School of Science and Engineering, Ehime University, Matsuyama 790-8577, JAPAN

E-mail address: dmitri.shakhmatov@ehime-u.ac.jp

Master's Course, Graduate School of Science and Engineering, Ehime University, Matsuyama 7908577, JAPAN

E-mail address: victor_yanez@comunidad.unam.mx 